\title{
The Factors Affecting Recurrence and Prognosis in Patients with Low-grade Stage Ta Bladder Cancer
}

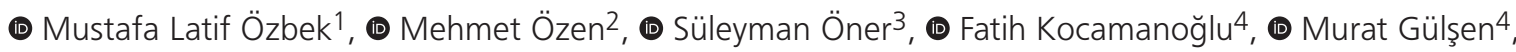 \\ (1) Mehmet Necmettin Mercimek ${ }^{5}$, (1) Yakup Bostancl${ }^{4}$, (1) Şaban Sarıkaya ${ }^{4}$ \\ 1 Private Atasam Hospital, Clinic of Urology, Samsun, Turkey \\ 2University of Health Scineces Turkey, Samsun Training and Research Hospital, Clinic of Urology, Samsun, Turkey \\ 3Yunus Emre State Hospital, Clinic of Urology, Eskişehir, Turkey \\ ${ }^{4}$ Ondokuz Mayıs University Faculty of Medicine, Department of Urology, Samsun, Turkey \\ 5 Samsun Liv Hospital, Clinic of Urology, Samsun, Turkey
}

\begin{abstract}
Objective: This study aims to review the parameters affecting tumour recurrence, tumour progression and cancer survival of patients with low-grade non-muscle invasive bladder cancer.

Materials and Methods: We retrospectively reviewed 262 patients with primary, low-grade Ta bladder cancer. Recurrence was defined as the occurrence of a new tumour in the prostatic urethra or bladder. Tumour progression was defined as confirmed high-grade Ta, all T1 or carcinoma in situ, upper tract recurrence or progression to T2. The associations between factors that affect recurrence and progression were analysed.

Results: Tumour recurrence and progression occurred in 119 (45.4\%) and 25 (9.5\%) patients during follow-up (median follow-up: $50.9 \pm 36.3$ months), respectively. Univariate and multivariate analyses demonstrate that smoking, multiple tumours and large tumours $(3 \mathrm{~cm}<)$ were significant. A Cox regression analysis revealed that progression was identified as a significant risk factor on survival. There was no effect of smoking on recurrence-free survival. A Kaplan-Meier analysis showed that one-, five- and ten-year progression-free survival rates were $99.6 \%, 88.2 \%$ and $70 \%$, respectively.

Conclusion: Multiple tumours, large tumours $(>3 \mathrm{~cm})$ and smoking were risk factors for recurrence and progression. Prevention of smoking and routine cystoscopic examination are essential in bladder cancer.
\end{abstract}

Keywords: Bladder cancer, progression, recurrence, risk factors

\section{Introduction}

Bladder cancer is a complex disease with high morbidity and mortality rates if it is left untreated. Approximately $75 \%-85 \%$ of bladder cancer is non-muscle invasive at the time of the diagnosis. Stage Ta tumours constitute approximately $70 \%$ of non-muscle invasive bladder cancers (NMIBC) at presentation. About 50\%-70\% of NMIBC relapsed and 5\%-30\% showed progression $(1,2)$.

Although stage Ta tumours are generally low grade, the highgrade disease rate has been $6.9 \%$. However, recurrence rates are high, especially in multiple tumours; progression is rare (3). Stage Ta tumours often relapse or progress after the first five years of treatment. The recurrence rate is approximately $50 \%-$ $60 \%$, and the progression rate is between $7 \%-20 \%$. Ta tumours usually show recurrence or progression within five years of initial treatment, but a longer follow-up (10-15 years) is needed $(4,5)$.
Low-grade stage Ta tumours rarely progress; however, they often recur. Therefore, knowing the patient's tumours characteristics, treatment and demographic characteristics may prevent recurrence and progression. This study aimed to review the parameters affecting recurrence and progression in patients with bladder cancer diagnosed with primary low-grade stage Ta.

\section{Materials and Methods}

This retrospective study has been approved by the Clinical Research Ethics Committee (2017/294), and all participants provided written informed consent to participate in this study. In this study, we retrospectively reviewed 308 patients who underwent transurethral bladder resection (TUR-B) between January 2009-January 2018 for initial diagnoses of low-grade stage Ta bladder tumours at least one year follow-up. The following factors were analysed for each individual from medical 
records: age, gender, smoking, systemic disease, tumour characteristics in cystoscopy (such as papillary, solid, multiple and solitary), tumour diameter in radiological image and time to progression.

Tumour stage was evaluated according to the 2009 American Joint Committee on Cancer tumor node metastasis classification, and tumour grade was evaluated according to $2004 \mathrm{WHO}$ classification (2).

Forty-six patients with a history of TUR-B in other centres, highgrade pathology, upper urinary tract cancer, outpatient followup and incomplete data in their medical records were excluded from this study. The surgical procedure was performed under spinal anaesthesia with bipolar or monopolar resectoscope after the patients' informed consent. In addition to the main tumour specimen obtained from TUR-B, the tumour base was also sampled and sent separately for histopathological examination. Small tumours $(<1 \mathrm{~cm})$ observed in cystoscopy were removed as en-block (2). The patients were hospitalised for an average of one day. The foley catheter was removed within a mean of two days. All patients received an intracavitary single dose of epirubicin/mitomycin within the first six hours. The patients' follow-up was performed with flexible cystoscopy at three or six months intervals for the first two years, and then every year later (2).

Radiological evaluation (computed tomography/magnetic resonance) was performed to detect upper urinary tract cancer or distant metastasis when positive urinary cytology, the suspicious invasive appearance was detected in the bladder. Recurrence was defined as the occurrence of a new tumour in the prostatic urethra or bladder and cured by transurethral resection. Progression of the tumour was defined as confirmed high-grade $\mathrm{Ta}$, all $\mathrm{T} 1$, or carcinoma in situ (CIS), upper tract recurrence, or progression to $\mathrm{T} 2$.

\section{Statistical Analysis}

Data were analysed with statistical software package IBM SPSS V23. Chi-square test was used to compare categorical data. Recurrence-free, progression-free survival analyses were performed using the Kaplan-Meier method. The log-rank test was applied to compare survival between a group of patients. Univariate regression, multivariate regression analysis and
Cox regression analysis were used to predict variables that affect recurrence and progression. The significance level was considered as $\mathrm{p}<0.05$.

\section{Results}

Of the 308 patients whose medical records were examined, 262 (72.4\%) were included in this study.

Of these, $92.4 \%$ (242) were male, and $7.6 \%$ (20) were female. The mean age was 67.58 \pm 10.1 years (range $38-92$ years), and 140 patients (53.4\%) had a history of smoking.

The presenting complaint was haematuria in 238 (90.8\%) cases and irritative symptoms in the remaining 24 patients. Twelve patients had diabetes mellitus, 16 had hypertension, four had chronic obstructive lung disease and three had coronary heart disease according to the medical records. During the follow-up period, a total of 15 patients (three of them due to metastatic bladder cancer) died. Ultrasonography and contrast/noncontrast tomography was used in preoperative radiological imaging.

A solitary tumour was detected in $162(61.8 \%)$ cases and multiple tumours in 100 (38.2\%).

In 49 patients (18.7\%), the tumour was larger than three $\mathrm{cm}$ (Table 1).

The mean follow-up period was $50.9 \pm 36.3$ months (range, 12-204). Recurrence was observed in 119 patients (45.4\%). The mean time to recurrence was 22.4 months (range, 2-176 months).

Univariate and multivariate analyses demonstrated that recurrence [Odds ratio (OR) $1.36695 \%$ confidence interval $(\mathrm{Cl})$, 0.389-4.801 $\mathrm{p}=0.021$, OR $0.62695 \% \mathrm{Cl}, 0.116-3.38 \mathrm{p}=0.036)]$ and progression (OR $5.76095 \% \mathrm{Cl}, 1.249-26.566, \mathrm{p}=0.007$, OR $8.31795 \% \mathrm{Cl}, 0.728-94.954 \mathrm{p}=0.017$ ) rates of the patients with a smoking history were significant. The recurrence rate was $75 \%$ in multiple tumours and $38.4 \%$ in solitary tumours. In the multivariate analysis, patients with multiple tumours, recurrence (OR 4.078, 95\% Cl 0.899-18.491, $\mathrm{p}<0.001$ ) and progression (OR 12.213, 95\% Cl 0.785-19.039, $\mathrm{p}<0.001$ ) rates were statistically significant compared with solitary tumours. Recurrence and progression $(p<0.001)$ rates were found to be significant in tumours larger than three $\mathrm{cm}$ compared with

Table 1. Clinical and demographic characteristics of patients

\begin{tabular}{|c|c|c|c|c|c|c|c|c|}
\hline \multicolumn{2}{|l|}{ Variable } & \multirow{2}{*}{$\begin{array}{l}\begin{array}{l}\text { Total } \\
\text { n (\%) }\end{array} \\
262 \\
\end{array}$} & \multirow{2}{*}{$\begin{array}{l}\text { Recurrence (+) } \\
119(45.4)\end{array}$} & \multirow{2}{*}{$\begin{array}{l}\text { Recurrence (-) } \\
143(54.6)\end{array}$} & \multirow[t]{2}{*}{$p$-value } & \multirow{2}{*}{$\begin{array}{l}\begin{array}{l}\text { Progression } \\
(+)\end{array} \\
25(9.5) \\
\end{array}$} & \multirow{2}{*}{$\begin{array}{l}\text { Progression } \\
(-)\end{array}$} & \multirow[t]{2}{*}{$p$-value } \\
\hline$n$ & & & & & & & & \\
\hline \multirow{2}{*}{ Gender } & Male & $242(92.4)$ & 113 & 129 & \multirow{2}{*}{0.143} & 23 & 212 & \multirow{2}{*}{0.736} \\
\hline & Female & $20(7.6)$ & 6 & 14 & & 2 & 18 & \\
\hline \multirow{2}{*}{ Smoking } & Yes & $140(53.4)$ & 72 & 68 & \multirow{2}{*}{0.035} & 19 & 121 & \multirow{2}{*}{0.002} \\
\hline & No & $122(46.6)$ & 47 & 75 & & 6 & 116 & \\
\hline \multirow{2}{*}{$\begin{array}{l}\text { Tumour } \\
\text { multiplicity }\end{array}$} & Solitary & $162(61.8)$ & 45 & 117 & \multirow{2}{*}{0.001} & 3 & 159 & \multirow{2}{*}{0.001} \\
\hline & Multiple & $100(38.2)$ & 74 & 26 & & 22 & 78 & \\
\hline \multirow{2}{*}{ Tumour size } & $>3 \mathrm{~cm}$ & 49 (18.7) & 27 & 22 & \multirow{2}{*}{0.021} & 1 & 48 & \multirow{2}{*}{0.001} \\
\hline & $3 \mathrm{~cm}$ & $213(81.3)$ & 79 & 134 & & 24 & 189 & \\
\hline
\end{tabular}




\begin{tabular}{|c|c|c|c|c|c|c|c|c|}
\hline & \multicolumn{4}{|l|}{ Recurrence } & \multicolumn{4}{|l|}{ Progression } \\
\hline & \multicolumn{2}{|l|}{ Univariate } & \multicolumn{2}{|l|}{ Multivariate } & \multicolumn{2}{|l|}{ Univariate } & \multicolumn{2}{|l|}{ Multivariate } \\
\hline & $\begin{array}{l}\text { OR } \\
(95 \% \mathrm{CI})\end{array}$ & $\mathbf{p}$ & $\begin{array}{l}\text { OR } \\
(95 \% \mathrm{Cl})\end{array}$ & $\mathbf{p}$ & $\begin{array}{l}\text { OR } \\
(95 \% \mathrm{Cl})\end{array}$ & $\mathbf{p}$ & $\begin{array}{l}\text { OR } \\
(95 \% \mathrm{Cl})\end{array}$ & $\mathbf{p}$ \\
\hline $\begin{array}{l}\text { Tumour } \\
\text { (multiple/solitary) }\end{array}$ & $\begin{array}{l}3.375 \\
(0.608-18.747)\end{array}$ & 0.057 & $\begin{array}{l}4.078 \\
(0.899-18.491)\end{array}$ & 0.001 & $\begin{array}{l}2.167 \\
(0.476-9.860)\end{array}$ & 0.055 & \begin{tabular}{|l}
12.213 \\
$(0.785-19.039$ \\
\end{tabular} & 0.001 \\
\hline $\begin{array}{l}\text { Gender } \\
\text { (male/female) }\end{array}$ & $\begin{array}{l}1.342 \\
(0.701-2.315)\end{array}$ & 0.918 & $\begin{array}{l}1.456 \\
(0.765-2.401)\end{array}$ & 0.151 & $\begin{array}{l}1.205 \\
(0.957-0.745)\end{array}$ & 0.674 & $0.915(0.608-0.455)$ & 0.942 \\
\hline $\begin{array}{l}\text { Smoking } \\
\text { (yes/no) }\end{array}$ & $\begin{array}{l}1.366 \\
(0.389-4.801)\end{array}$ & 0.021 & $0.626(0.116-3.38)$ & 0.036 & $\begin{array}{l}5.760 \\
(1.249-26.566)\end{array}$ & 0.007 & $\begin{array}{l}8.317 \\
(0.728-94.954)\end{array}$ & 0.017 \\
\hline $\begin{array}{l}\text { Tumour size } \\
(<3 \mathrm{~cm} />3 \mathrm{~cm})\end{array}$ & $\begin{array}{l}3.879 \\
(0.908-16.747\end{array}$ & 0.001 & $\begin{array}{l}5.078 \\
(0.1899-16.444)\end{array}$ & 0.001 & $\begin{array}{l}5.167 \\
(0.976-11.538)\end{array}$ & 0.001 & $\begin{array}{l}13.213 \\
(0.989-21.011\end{array}$ & 0.001 \\
\hline
\end{tabular}

tumours smaller than three $\mathrm{cm}$. According to the multivariate analysis, gender was not an influential factor in patients' recurrence and progression (Table 2).

According to the Kaplan-Meier survival analysis, recurrencefree survival at one, five and ten years was 98\%, 65.2\% and $27 \%$, respectively. The 5-year recurrence-free survival in multiple tumours was $46 \%$ and $71 \%$ in solitary tumours ( $p=0.003$ ). There was no effect of smoking on recurrence-free survival (Figures $1,2)$.

There was no statistically significant difference between the mean survival times, according to recurrence $(p=0.177)$ (Figure 1). The mean survival time was 176.76 months in patients with recurrence and 150.27 months in patients without recurrence. A total of 25 patients (9.5\%) had progression, and the time to progression was $27.3 \pm 25.2$ months (range, 6-110 months). Both univariate and multivariate analyses demonstrated that smoking and multiple tumours had a high progression rate $(p=0.017$, $\mathrm{p}<0.001)$. The Kaplan-Meier analysis showed that one-, fiveand ten-year progression-free survival rates were $99.6 \%, 88.2 \%$ and $70 \%$, respectively. When the factors affecting the KaplanMeier 5-year progression-free survival were examined, it was found that only the multiple tumours (79\%) were compared with the solitary tumours $(98 \%)(p=0.003)$ (Figures 3,4$)$.

The Cox regression analysis revealed that smoking, tumour characteristics, and recurrence status were not independent risk factors. Progression status was identified as a significant risk factor on survival, and the presence of progression increased the mortality risk by 18.018 times compared with non-progression $(p<0.001)$. The Cox regression analysis is shown in Table 3.

Recurrence was observed in three patients, progression was observed in one of 23 patients who had been followed for more than 10 years.

Recurrence was observed in three patients, progression was observed in one of 23 patients who had been followed for more than 10 years. High-grade Ta/T1, T2-T4, CIS, Upper urinary tract tumour progression rates were 13 (4.9\%), 8 (3\%), $2(0.7 \%), 2$ $(0.7 \%)$, respectively. Progression significantly affected the mean survival time. The mean survival was 101.48 months in patients with progression and 200.19 months in patients without progression $(p<0.001)$. Survival graphs are presented in Figure 1 and Figure 3.

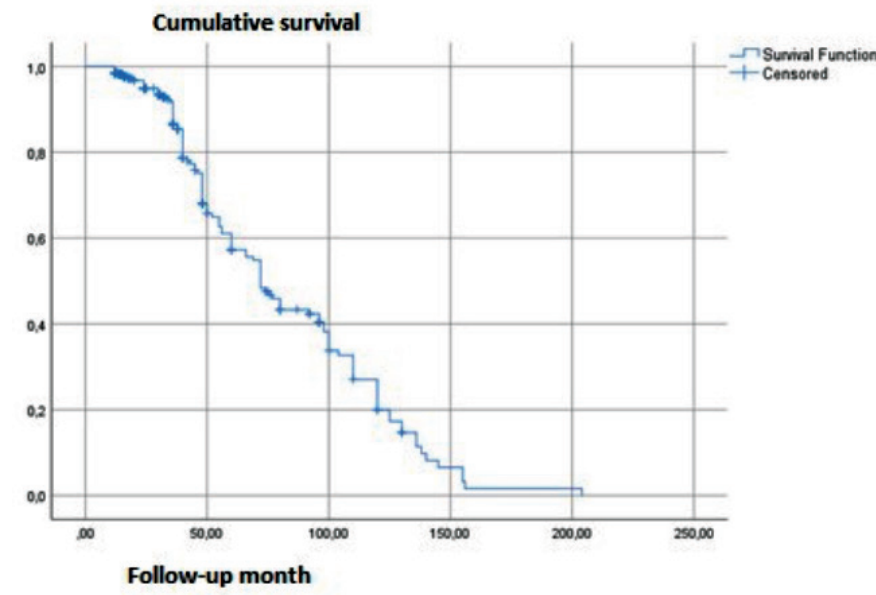

Figure 1. Reccurence-free survival rates, reccurence-free survival 1 and 5 years; $98 \%, 65.2 \%$

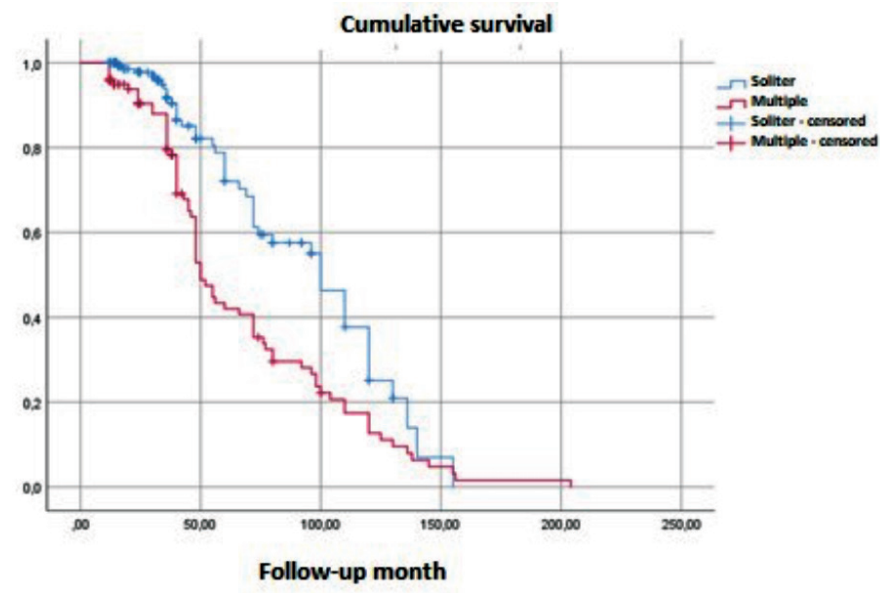

Figure 2. Reccurence-free survival rates in multiple \& soliter tumors, reccurencefree survival rates soliter tumors $71 \%$ and multiple tumors $46 \%$

\section{Discussion}

This study is a retrospective review of 262 patients with primary low-grade stage Ta bladder cancer. Patient-related parameters that could affect recurrence and progression rates (age, sex, smoking, tumour characteristics) were analysed. Multivariate 


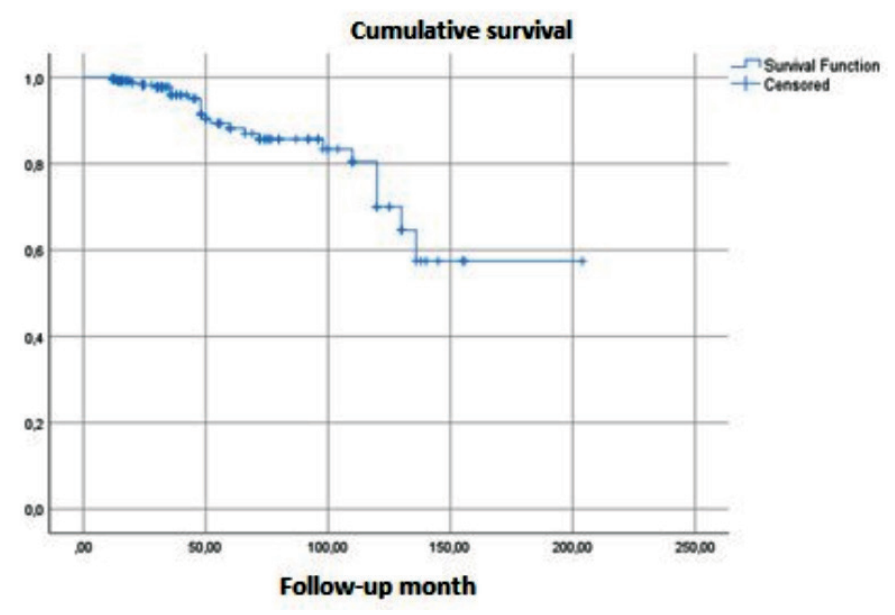

Figure 3. Progression-free survival rates, progression-free survival 1 and 5 years $99.6 \%, 88.2 \%$

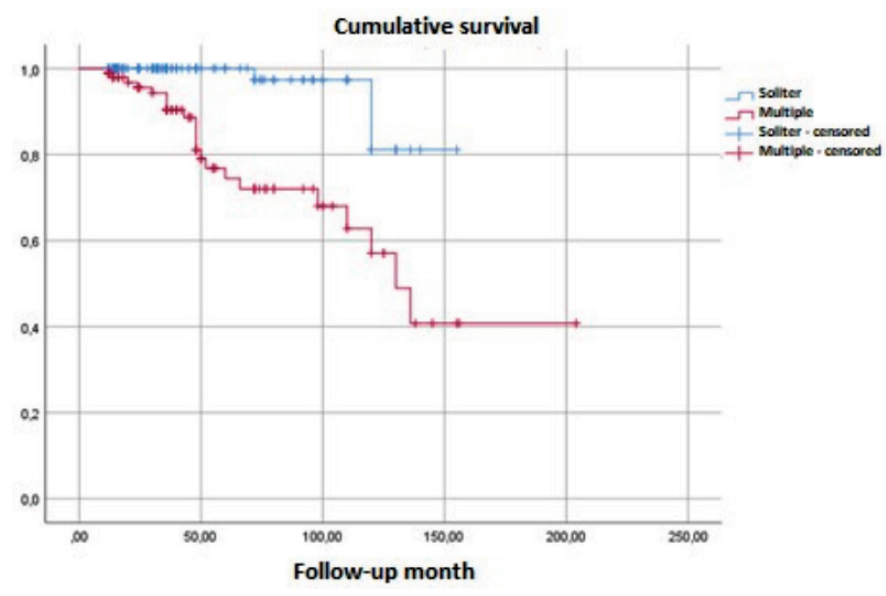

Figure 4. Progression-free survival rates in multiple \& soliter tumors, progressionfree survival rates multiple tumors $79 \%$, soliter tumors $98 \%$

\begin{tabular}{|l|l|l|}
\hline \multicolumn{4}{|l|}{ Table 3. Cox regression analyses } \\
\hline & OR $(95 \% \mathrm{Cl})$ & $\mathbf{p}$ \\
\hline Smoking (yes/no) & $3.255(0.979-10.826)$ & 0.054 \\
\hline Tumour (multiple/solitary) & $1.994(0.547-7.266)$ & 0.295 \\
\hline Tumour size $(\leq 3 \mathrm{~cm},>3 \mathrm{~cm})$ & $2.108(0.653-5.615)$ & 0.145 \\
\hline Recurrence (yes/no) & $0.945(0.217-4.114)$ & 0.940 \\
\hline Progression (yes/no) & $18.018(4.804-67.581)$ & $<0.001^{*}$ \\
\hline *Statistically significant $\mathrm{p}<0.05$, OR: Odds ratio, Cl: Confidence interval \\
\hline
\end{tabular}

analysis showed a high risk of recurrence and progression in patients with multiple focal tumours and smokers. While solitary tumours had a risk of recurrence of less than $48 \%$, multiple stage Ta tumours had a risk of approximately 50\%-70\% $(5,6)$. In Golabesk et al.'s (4) single-centre retrospective study, 704 NMBC Ta/G1-G2 T1/G1-G2 patients were followed up for a mean of 64.9 months. Recurrence was observed in 188 (34.3\%) of 548 Ta patients. Rieken et al. (5), in their multicentre retrospective analysis, 1436 low-grade Ta-stage patients were divided into two groups as low and moderate risk. After a mean follow-up of 33.5 months, recurrence was found in 613 patients (42.7\%), and 5-year recurrence-free survival was 56\%. According to the Cox regression analysis, advanced age, tumour size larger than three $\mathrm{cm}$ and recurrence rate of multiple tumours were significant. In this study, progression was observed in 164 patients (11.4\%) after a mean follow-up of 67.2 months. However, the 5-year progression-free survival was $95 \%$ (5).

In a single-centre retrospective analysis conducted by Kobayashi et al. (7), 190 patients with low-grade Ta were evaluated for long-term, tumour recurrence was $43.2 \%$, and progression was $11.1 \%$ in 101.5 months. Multiple tumour and intravesical instillation were risk factors for recurrence in multivariate analysis, whereas multiple tumour progression was the only risk factor for recurrence. After five years of tumour-free survival, nine patients had a late recurrence, and 10 years later, two patients had late recurrence after gross haematuria. Similarly, Matsumoto et al. (8) observed recurrence in 39 patients (14.9\%) and progression in five patients $(1.9 \%)$ during the follow-up of patients who did not have a 5-year recurrence in their retrospective studies.

Our study was consistent with Kobayashi (7) and Matsumoto's (8) findings since 10 patients (3.8\%) had a late relapse after five years of recurrence, and two $(0.76 \%)$ patients had late progression. There were 23 patients whose follow-up periods were over 10 years, three patients had a recurrence, and one patient had progression. To our knowledge, although there are no studies described in the literature for late recurrence, it is recommended that low-grade Ta tumours should be under routine follow-up for up to 10 years. However, there are also publications in the literature indicating that follow-up with cystoscopy is unnecessary in low-risk bladder cancer (9).

In Nerli et al.'s (10) single-centre retrospective study, 42 patients with low-grade, multiple, stage Ta bladder cancer were followed for a mean of $57.38 \pm 28.4$ months (range 12 to 118 months). Recurrence was seen in 23 patients (54.76\%) and progression in eight patients (19.04\%) (10). In our study, the recurrence rate was $74 \%$, and the progression rate was $22 \%$ in multiple tumours. In a multivariate analysis, the recurrence rate was significant in smoking patients, and 5-year relapse-free survival was significantly higher than in non-smokers $(74 \%-42.5 \%$, $\mathrm{p}=0.0001$ ).

In Zieger et al.'s (11) a single-centre prospective study, 212 patients with stage Ta G1-2 tumours were followed for 20 years, and only 14 patients received intravesical treatment. Ten patients (4.7\%) had TaG3 CIS, 18 (8.5\%) had stage T1, 23 patients $(10.8 \%)$ had muscle invasion and distant metastasis (11). Similarly, Prout et al. (12) followed 178 stage Ta G1 patients for 10 years and observed progression in 13 patients (7.3\%). In Akagashi et al.'s (13) study, none of the patients with TaG1-2, muscle invasion were not observed, 9.7\% T1 and CIS progression were observed, and patients received intravesical treatment for two years. Also, in our study, the progression rate was $9.5 \%$, which is consistent with the literature.

In the study of 245 low-grade Ta patients selected in the lowrisk group in 2018 regarding the number and size of tumours, it was concluded that $1.5 \mathrm{~cm}$ might be the cut-off value. It also reported a higher recurrence rate of eight or more tumours (14). Also, in our study, the recurrence rates were higher in tumours larger than $3 \mathrm{~cm}$. Tobacco use is a known risk factor for bladder 
cancer. There are publications in the literature that tobacco use increases recurrence rates. In a retrospective analysis published in 2017, patients were categorised as non-smokers, smokers and former smokers. It is suggested that high cumulative exposure may increase smokers' recurrence rate, whereas people who stop smoking may have lower recurrence rates than smokers (15). Our study shows that recurrence and progression rates were significantly higher in smokers.

Although it is not included in the EAU and AUA guidelines, in recent years, as in prostate cancer, active monitoring has been conducted in NMIBC. In the study conducted in 181 cases followed by active surveillance in 2018, recurrence was observed in $61(33.7 \%), 20$ had benign lesions, and 41 (22.6\%) had tumour recurrence. The authors suggest that active monitoring may be performed in NMIBC (16). Further prospective studies on active surveillance are needed.

\section{Study Limitations}

There are some limitations to our study. Since our study is retrospective, there may be incorrect and conflicting information in the medical records. Conflicting and incomplete data are excluded from this study as much as possible. Second, we could not find any information in the patient medical records regarding smoking behaviour, such as how many packs of cigarettes smoked per day, how many years they smoked. Third, it is stated in the literature that the recurrence rate decreased in people who receive postoperative intracavitary chemotherapy (ICT) (17). However, all of our patients received postoperative ICT, and there was no control group, so no comparison was made. Similarly, patients with high-risk bladder tumours who received ICT and BCG treatment were not in the control group, and those who received ICT treatment were statistically insignificant. Therefore, we believe that prospective comparative studies with high patient numbers will provide more accurate results.

\section{Conclusion}

In our study, recurrence and progression rates were $45.4 \%$ and $9.5 \%$ in primary low-grade Ta tumours, respectively. Smoking, tumours larger than three $\mathrm{cm}$ and multiple tumours increase the risk of recurrence and progression. Therefore, the prevention of smoking and routine cystoscopic examination is essential in the diagnosis and treatment of bladder cancer.

\section{Acknowledgements}

Publication: The results of the study were not published in full or in part in form of abstracts.

Contribution: There is not any contributors who may not be listed as authors.

Conflict of Interest: No conflict of interest was declared by the authors.

Financial Disclosure: The authors declared that this study received no financial support.

\section{Ethics}

Ethics Committee Approval: This retrospective study has been approved by the Clinical Research Ethics Committee (2017/294).

Informed Consent: All participants provided written informed consent to participate in this study.
Peer-review: Externally and internally peer-reviewed.

\section{Authorship Contributions}

Concept: M.L.Ö., M.N.M., Design: M.L.Ö., Data Collection or Processing: M.Ö., S.Ö., F.K., M.G., Analysis or Interpretation: Y.B., Ş.S., Literature Search: M.L.Ö., Writing: M.L.Ö.

\section{References}

1. Soukup V, Capoun O, Cohen D, et al. Prognostic Performance and Reproducibility of the 1973 and 2004/2016 World Health Organization Grading Classification Systems in Non-muscle-invasive Bladder Cancer: A European Association of Urology Non-muscle Invasive Bladder Cancer Guidelines Panel Systematic Review. Eur Urol 2017;72:801-813.

2. Babjuk M, Burger M, Comperat EM, et al. European Association of Urology Guidelines on Non-muscle-invasive Bladder Cancer (TaT1 and Carcinoma In Situ) - 2019 Update. Eur Urol 2019;76:639-657.

3. Sylvester RJ, van der Meijden A, Witjes JA, et al. High-grade Ta urothelial carcinoma and carcinoma in situ of the bladder. Urology 2005;66(6 Suppl 1):90-107.

4. Golabesk T, Palou I, Rodriguez $\mathrm{O}$, et al. Long-term Bladder and Upper Urinary Tract Follow-up Recurrence and Progression Rates of G1-2 Non-muscle-invasive Urothelial Carcinoma of the Bladder. Urology 2017;100:145-150.

5. Rieken $M$, Xylinas $E$, Kluth $L$, et al. Long-term cancer-specific outcomes of TaG1 urothelial carcinoma of the bladder. Eur Urol 2014;65:201-209.

6. Herr HW. Tumor progression and survival of patients with high grade, noninvasive papillary (TaG3) bladder tumors: 15-year outcome. J Urol 2000;163:60-61; discussion 61-62.

7. Kobayashi $H$, Kikuchi E, Mikami S, et al. Long term follow-up in patients with initially diagnosed low grade Ta non-muscle invasive bladder tumors: tumor recurrence and worsening progression. BMC Urol 2014;14:5.

8. Matsumoto $\mathrm{K}$, Kikuchi $\mathrm{E}$, Horiguchi $\mathrm{Y}$, et al. Late recurrence and progression in non-muscle-invasive bladder cancers after 5-year tumorfree periods. Urology 2010;75:1385-1390.

9. Han DS, Lynch KE, Chang JW, et al. Overuse of Cystoscopic Surveillance Among Patients With Low-risk Non-Muscle-invasive Bladder Cancer - A National Study of Patient, Provider, and Facility Factors. Urology 2019;131:112-119.

10. Nerli RB, Ghagane SC, Shankar K, et al. Low-Grade, Multiple, Ta Nonmuscle-Invasive Bladder Tumors: Tumor Recurrence and Worsening Progression. Indian J Surg Oncol 2018;9:157-161.

11. Zieger K, Wolf $\mathrm{H}$, Olsen PR, Hojgaard K. Long-term follow-up of noninvasive bladder tumours (stage Ta): recurrence and progression. BJU Int 2000;85:824-828.

12. Prout GR Jr, Barton BA, Griffin PP, Friedell GH. Treated history of noninvasive grade 1 transitional cell carcinoma. The National Bladder Cancer Group. J Urol 1992;148:1413-1419.

13. Akagashi K, Tanda H, Kato $S$, et al. Recurrence pattern for superficial bladder cancer. Int J Urol 2006;13:686-691.

14. Akitake M, Kiyoshima K, Yokomizo A, et al. A rational risk assessment for intravesical recurrence in primary low-grade Ta bladder cancer: A retrospective analysis of 245 cases. Mol Clin Oncol 2018;8:785-790.

15. Li HM, Azhati B, Rexiati M, et al. Impact of smoking status and cumulative smoking exposure on tumor recurrence of non-muscle-invasive bladder cancer. Int Urol Nephrol 2017;49:69-76.

16. Hurle R, Colombo P, Lazzeri M, et al. Pathological Outcomes for Patients Who Failed to Remain Under Active Surveillance for Low-risk Non-muscleinvasive Bladder Cancer: Update and Results from the Bladder Cancer Italian Active Surveillance Project. Eur Urol Oncol 2018;1:437-442.

17. Sylvester RJ, Oosterlinck W, van der Meijden AP. A single immediate postoperative instillation of chemotherapy decreases the risk of recurrence in patients with stage Ta T1 bladder cancer: a meta-analysis of published results of randomized clinical trials. J Urol 2004;171(6 Pt 1):2186-2190, quiz 2435. 